\title{
Intervención clínica sobre los principales factores de riesgo vascular (estudio RIVANA)
}

\section{Clinical interventions on the major vascular risk factors (RIVANA study)}

\author{
C. Amézqueta ${ }^{1}$, B. Bermejo ${ }^{2}$, M.J. Guembe ${ }^{3}$, M.T. Artázcoz ${ }^{4}$, J.J. Viñes ${ }^{3}$
}

\section{RESUMEN}

Fundamento. El objetivo es describir la frecuencia de diagnóstico de los factores clásicos de riesgo vascular y de las intervenciones dietéticas y terapéuticas, así como su control.

Sujetos y métodos. Dentro del proyecto Riesgo Vascular de Navarra (RIVANA), se lleva a cabo el estudio transversal en una muestra aleatoria de 4.354 personas entre 35 y 84 años, recogiendo por encuesta antecedentes y realizando exploración clínica y analítica. Los sujetos se han clasificado para cada factor de riesgo en: diagnosticados por información cuando el personal sanitario le había informado de que presentaba el factor de riesgo-; por intervención -cuando toma medicación para ello-; y por detección en personas asintomáticas. Se identifican las variables de intervención por árboles dicotómicos expresadas por frecuencias relativas.

Resultados. El 45,3\% de los sujetos son hipertensos: $27 \%$ diagnosticados previamente y $18,3 \%$ en el momento de la exploración. El 39\% son hipercolesterolémicos: 33,4\% diagnosticados previamente y $5,6 \%$ en el momento de la exploración. El 21,2\% presentaban hiperglucemia: 9,7\% diagnosticados previamente y $11,6 \%$ en el momento de la exploración. El $65,6 \%$ fue diagnosticado de sobrepeso u obesidad: $25,9 \%$ previamente y el 39,6\% en el momento de la exploración.

Recibían consejo dietético y tratamiento farmacológico respectivamente: el $79,8 \%$ y el $70,3 \%$ de los hipertensos; el $75,4 \%$ y el $35 \%$ de los hipercolesterolémicos; el $83,1 \%$ y $47,4 \%$ de los que presentaban hiperglucemia y el $68,5 \%$ y $4 \%$ de los que presentaban sobrepeso u obesidad.

Conclusiones. La frecuencia de los factores de riesgo cardiovascular es elevada. Existe un margen de mejora importante en la detección, intervención y control de los factores de riesgo.

Palabras clave. Factores de riesgo vascular. Prevalencia. Consejo dietético. Tratamiento farmacológico.

\begin{abstract}
Background. To describe the diagnostic frequency of classical vascular risk factors, dietary and therapeutic interventions and their control.

Methods. Within the project Vascular Risk in Navarre (RIVANA), a transversal study was made of a random sample of 4,354 people between the ages of 35 and 84 ; antecedents were gathered by interview and clinical and analytical exploration was carried out. The subjects were classified for each risk factor into: diagnosed by information -when health personnel had informed them that they presented a risk factor-; by intervention -when they take medication for it-; and by detection in asymptomatic people. The variables of intervention were identified by dichotomic trees expressed by relative frequencies.
\end{abstract}

Results. Forty-five point three percent of the subjects are hypertense: $27 \%$ previously diagnosed and $18.3 \%$ at the time of the exploration. Thirty-nine percent are hypercholesterolemic: $33.4 \%$ previously diagnosed and $5.6 \%$ at the time of the exploration. Twenty-one point two percent presented hyperglycemia: $9.7 \%$ previously diagnosed and $11.6 \%$ at the time of the exploration. Sixty-five point six percent were diagnosed as overweight or obese: $25.9 \%$ previously and $39.6 \%$ at the time of the exploration.

They received dietary counselling and pharmacological treatment respectively: $79.8 \%$ and $70.3 \%$ of the hypertense; $75.4 \%$ and $35 \%$ of the hypercholesterolemic; $83.1 \%$ and $47.4 \%$ of those who presented hyperglycemia and $68.5 \%$ and $4 \%$ of those who showed overweight or obesity.

Conclusions. The frequency of cardiovascular risk factors is high. There is a margin for significant improvement in the detection, intervention and control of the risk factors.

Key words. Vascular risk factors. Frequency. Dietary advice. Drug treatment.
1. Servicio Navarro de Salud. Centro de Salud Iturrama. Dirección de Atención Primaria (Estudio RIVANA).

2. Servicio de Docencia y Desarrollo Sanitarios. Departamento de Salud. Gobierno de Navarra.

3. Servicio de Docencia y Desarrollo Sanitarios. Departamento de Salud. Gobierno de Navarra (Estudio RIVANA).

4. Servicio de Prestaciones Farmacéuticas. Servicio Navarro de Salud.

Los autores declaran que no están inmersos en conflicto de intereses. El Proyecto RIVANA ha sido aprobado por el Comité Ético de Investigación Clínica de la Comunidad Foral de Navarra y se ajusta a las normas de Helsinki

Proyecto financiado por el Instituto de Salud Carlos III por Convenio con el Gobierno de Navarra de fecha 26 de diciembre de 2003 y por los presupuestos generales de Navarra de 2004 y 2005

\section{Correspondencia}

Carlos Amézqueta Goñi

Centro de Salud de Iturrama

Servicio Navarro de Salud

Serafin Olave $\mathbf{s} / \mathbf{n}$

31007 Pamplona

Tfno. 848429315

Fax 848428567

E-mail: camezqug@cfnavarra.es

Recepción: 1 de abril de 2009

Aceptación provisional: 7 de mayo de 2009

Aceptación definitiva: 1 de septiembre de 2009 


\section{INTRODUCCIÓN}

En Navarra las muertes causadas por enfermedades vasculares ocupan, desde el año 2001, el segundo lugar en hombres, tras los procesos oncológicos y el primero en mujeres. Las tasas de mortalidad por enfermedad isquémica del corazón y por enfermedad cerebrovascular, sin embargo, han disminuido en el periodo 2001-2005 más del $10 \%$ en ambos sexos respecto al periodo 1996-2000. Este hecho mantiene a Navarra entre las regiones de Europa con tasas más bajas y contribuye al incremento observado en la esperanza de vida ${ }^{1}$.

Estudios realizados en Inglaterra y Ga$\operatorname{les}^{2}$ y en EEUU ${ }^{3}$ atribuyen al menos la mitad de la reducción en la mortalidad coronaria al mejor control de los factores de riesgo relacionados.

En los últimos años se han desarrollado en Navarra estrategias poblacionales e individuales con el objetivo de intensificar la prevención cardiovascular, pero no se conoce suficientemente la implantación o el cumplimiento en la práctica clínica de las medidas propuestas ${ }^{4}$.

El presente trabajo tiene como objetivo describir la frecuencia con la que en la clínica son identificados los factores de riesgo cardiovascular clásicos y la intervención dietética y terapéutica, así como el seguimiento y control realizados en los pacientes. El tabaco, siendo un hábito y factor clásico relevante, tiene características diferenciadas de identificación, intervención y control, por lo que es objeto de estudio y publicación específica, no incluyéndose en el presente artículo.

\section{SUJETOS Y MÉTODOS}

El trabajo se encuadra en el estudio Riesgo Vascular de Navarra (RIVANA) de carácter transversal, aleatorio con una muestra de 6.553 personas (3.088 hombres y 3.465 mujeres) entre 35 y 84 años, proporcionalmente por edad y sexo, de 24 zonas básicas de salud de Navarra ${ }^{5}$. El tamaño de la muestra se calculó en función de la menor prevalencia estimada de síndrome metabólico, según estudio de los factores de riesgo en Navarra de 1993 (7\%), con una precisión del $10 \%$ y un nivel de confianza del $95 \%{ }^{6}$.

Se pasó un cuestionario obteniendo una participación del 76\% (4.354 personas) y datos completos de encuesta, exploración y analítica del 73,4\% (4.168 personas) ${ }^{7}$.

En el cuestionario, se solicitaban, además de los datos de filiación, edad y sexo, los siguientes datos para cada uno de los factores de riesgo:

- Si había sido informado por el personal sanitario de su diagnóstico.

- Si el personal sanitario le aconsejó seguir la dieta correspondiente.

- Si en las dos últimas semanas tomó algún medicamento para el factor de riesgo diagnosticado (se adjuntaban los envases).

- Si se realizó una medición o analítica, en el último año, de dichos factores de riesgo.

La exploración física para la determinación de sobrepeso, obesidad (IMC), presión arterial (PA) y determinación de valores analíticos se ajustó a la metodología del estudio de Riesgo Vascular de Navarra (RIVANA) ${ }^{7}$. Éste incluyó la medida del perímetro abdominal a todas las personas. La medición de presión arterial se realizó con aparatos automáticos validados (OMRON M 4-1).

\section{Definición de los factores de riesgo y clasificación diagnóstica}

Se consideró persona hipertensa cuando cumplía alguno de los siguientes criterios:

- Haber sido informado previamente por personal sanitario de tener hipertensión arterial.

- No referir haber sido diagnosticado, pero tomar tratamiento farmacológico para la hipertensión.

- Presentar en el estudio cifras medias de PAS $\geq 140 \mathrm{mmHg}$ o de $\mathrm{PAD} \geq 90$ $\mathrm{mmHg}$. 
Se considera persona con hipercolesterolemia definida cuando cumplía alguno de los siguientes criterios:

- Haber sido informado por personal sanitario de tener hipercolesterolemia.

- No referir haber sido diagnosticado, pero tomar tratamiento farmacológico hipocolesteremiante.

- Presentar en el estudio cifras de colesterol total igual o superior a $250 \mathrm{mg} / \mathrm{dl}$.

Se considera sujeto con alteración de glucemia o diabetes si cumplía con alguno de los siguientes criterios:

- Haber sido informado de que presenta elevación del azúcar o de que es diabético.

- No referir haber sido diagnosticado, pero tomar tratamiento farmacológico para la diabetes.

- Presentar la glucemia basal alterada (entre 110 y $125 \mathrm{mg} / \mathrm{dl}$ ) o diabetes (glucemia $\geq 126 \mathrm{mg} / \mathrm{dl}$ ).

Se considera persona con sobrepeso u obesidad si:

- Refiere haber sido informado de tener sobrepeso o ser obeso.

- No refiere haber sido diagnosticado, pero toma tratamiento farmacológico para la obesidad.

- Presenta sobrepeso (IMC $\geq 25 \mathrm{~kg} / \mathrm{m}^{2} \mathrm{y}$ $<30 \mathrm{~kg} / \mathrm{m}^{2}$ ) u obesidad (IMC $\geq 30 \mathrm{~kg} / \mathrm{m}^{2}$ ).

Se considera obesidad abdominal si presenta un perímetro $>102 \mathrm{~cm}$ en los hombres $\mathrm{y}>88 \mathrm{~cm}$ en las mujeres.

\section{Clasificación de intervención y control clínicos}

Los pacientes diagnosticados por factores de riesgo se clasificaron en cuatro grupos en función de la intervención: sin tratamiento, solo dieta, dieta y medicación y exclusivamente medicación.

Para cada uno de estos grupos se determinó cuántos pacientes tenían, en el momento de la encuesta, el factor de riesgo en situación de control. Se consideraron controlados cuando estando diagnosticados y en tratamiento, mantenían los siguientes niveles: PAS $<140$ $\mathrm{mmHg}$ y $\mathrm{PAD}<90 \mathrm{mmHg}$, colesterol total inferior a $250 \mathrm{mg} / \mathrm{dl}$, glucemia $\leq 130 \mathrm{mg} / \mathrm{dl}$, $\mathrm{IMC}<25 \mathrm{~kg} / \mathrm{m}^{2}$.

Por último, para cada uno de los grupos se recogió el porcentaje de personas que en la encuesta refirieron haberse realizado alguna medición del factor de riesgo en estudio en el último año. Estas mediciones se denominan en las figuras como "revisiones".

\section{RESULTADOS}

La frecuencia de los factores de riesgo (Tabla 1), la frecuencia de personas diagnosticadas en el momento de la exploración (Tabla 2) y la frecuencia de las intervenciones sobre los factores de riesgo (Tabla 3) se presentan a continuación.

Tabla 1. Frecuencia diagnóstica de los factores de riesgo vascular según el sexo en población de 35 a 84 años. Muestra de 4.354 personas (2.370 mujeres y 1.984 hombres) para hipertensión y obesidad y de 4.168 (2.275 mujeres y 1.893 hombres) para hipercolesterolemia e hiperglucemia (disponibilidad analítica).

\begin{tabular}{|l|c|c|c|c|}
\hline & Hipertensión & Hipercolesterolemia & Hiperglucemia & $\begin{array}{c}\text { Sobrepeso y } \\
\text { obesidad }\end{array}$ \\
\hline Hombres & $1.052(53,1 \%)$ & $778(41,1 \%)$ & $532(28,1 \%)$ & $1.532(77,3 \%)$ \\
\hline Mujeres & $920(38,8 \%)$ & $848(37,3 \%)$ & $359(15,8 \%)$ & $1.319(55,7 \%)$ \\
\hline Global & $1.972(45,3 \%)$ & $1.626(39,0 \%)$ & $891(21,4 \%)$ & $2.851(65,5 \%)$ \\
\hline
\end{tabular}


Tabla 2. Frecuencia diagnóstica de los factores de riesgo vascular según el momento del diagnóstico: previo referido por el paciente $v s$ realizado tras estudio. Calculados sobre una muestra de 4.354 personas para hipertensión y obesidad y de 4.168 para hipercolesterolemia e hiperglucemia (analítica disponible).

\begin{tabular}{|l|c|c|c|c|}
\hline & Hipertensión & Hipercolesterolemia & Hiperglucemia & $\begin{array}{c}\text { Sobrepeso y } \\
\text { obesidad }\end{array}$ \\
\hline Diagnóstico previo & $1.175(27,0 \%)$ & $1.391(33,4 \%)$ & $409(9,7 \%)$ & $1.130(25,9 \%)$ \\
\hline $\begin{array}{l}\text { Diagnóstico en } \\
\text { el momento del } \\
\text { estudio }\end{array}$ & $797(18,3 \%)$ & $235(5,6 \%)$ & $482(11,6 \%)$ & $1.721(39,5 \%)$ \\
\hline Global & $1.972(45,3 \%)$ & $1.626(39,0 \%)$ & $891(21,4 \%)$ & $2.851(65,5 \%)$ \\
\hline
\end{tabular}

Tabla 3. Intervención en factores de riesgo: consejo dietético y prescripción farmacológica en sujetos diagnosticados previamente y en población global.

\begin{tabular}{|c|c|c|c|c|}
\hline & Hipertensión & Hipercolesterolemia & Hiperglucemia & $\begin{array}{c}\text { Sobrepeso/ } \\
\text { Obesidad }\end{array}$ \\
\hline $\begin{array}{l}\text { Pacientes } \\
\text { diagnosticados }\end{array}$ & 1.175 & 1.391 & 409 & 1.130 \\
\hline Consejo dietético & $938(79,8) \%$ & $1.048(75,4 \%)$ & $340(83,1 \%)$ & $774(68,5 \%)$ \\
\hline Medicación & $826(70,3) \%$ & $487(35,0 \%)$ & $194(47,4 \%)$ & $45(4,0 \%)$ \\
\hline Muestra población & 4.354 & 4.168 & 4.168 & 4.354 \\
\hline Medicación & $826(18,9 \%)$ & $487(11,7 \%)$ & $194(4,7 \%)$ & $45(1,0 \%)$ \\
\hline
\end{tabular}

\section{Hipertensión arterial}

Sobre las 4.354 personas exploradas, la frecuencia de HTA fue el $45,3 \%$ (53,1\% en hombres y $38,8 \%$ en mujeres). Del total de personas hipertensas identificadas, el $57,4 \%$ refieren estar previamente diagnosticados; el 2,2\%, aún no se reconocen diagnosticados como hipertensos, pero tienen indicado un fármaco hipotensor en su tratamiento habitual. El 40,4\% de los sujetos, no estaban diagnosticados previamente, presentando cifras medias superiores a 140/90 mm Hg en el momento de la exploración (Fig. 1).

Del conjunto de personas previamente diagnosticadas, el $62,1 \%$ informa que se le había indicado medicación y dado consejo dietético. El $17,7 \%$ ha recibido exclusivamente indicaciones dietéticas y un $8,2 \%$ únicamente prescripción farmacológica. El $12 \%$ refiere no haber recibido consejo die- tético ni tratamiento con medicación específica para su HTA.

El 32,9\% de los hipertensos ya diagnosticados presentan cifras medias de PA inferiores a 140/90 $\mathrm{mmHg}$. El control varía según el tipo de intervención, desde el 31,1\% de los pacientes a los que se les había indicado dieta y medicación, hasta el $43,8 \%$ en los que afirman que únicamente se les indicó medicación. Presentan cifras de control correcto (cifras inferiores a los niveles diagnósticos), el 36,9\% de los hipertensos diagnosticados que no refieren que se les haya indicado tratamiento alguno.

El 94,6\% de los hipertensos previamente diagnosticados afirmaban que se habían tomado la tensión arterial en el último año. En los hipertensos no diagnosticados, el 69,6\% reconoce haberse tomado la PA en el último año, porcentaje similar al de la población no hipertensa, que alcanza el $66,7 \%$. 


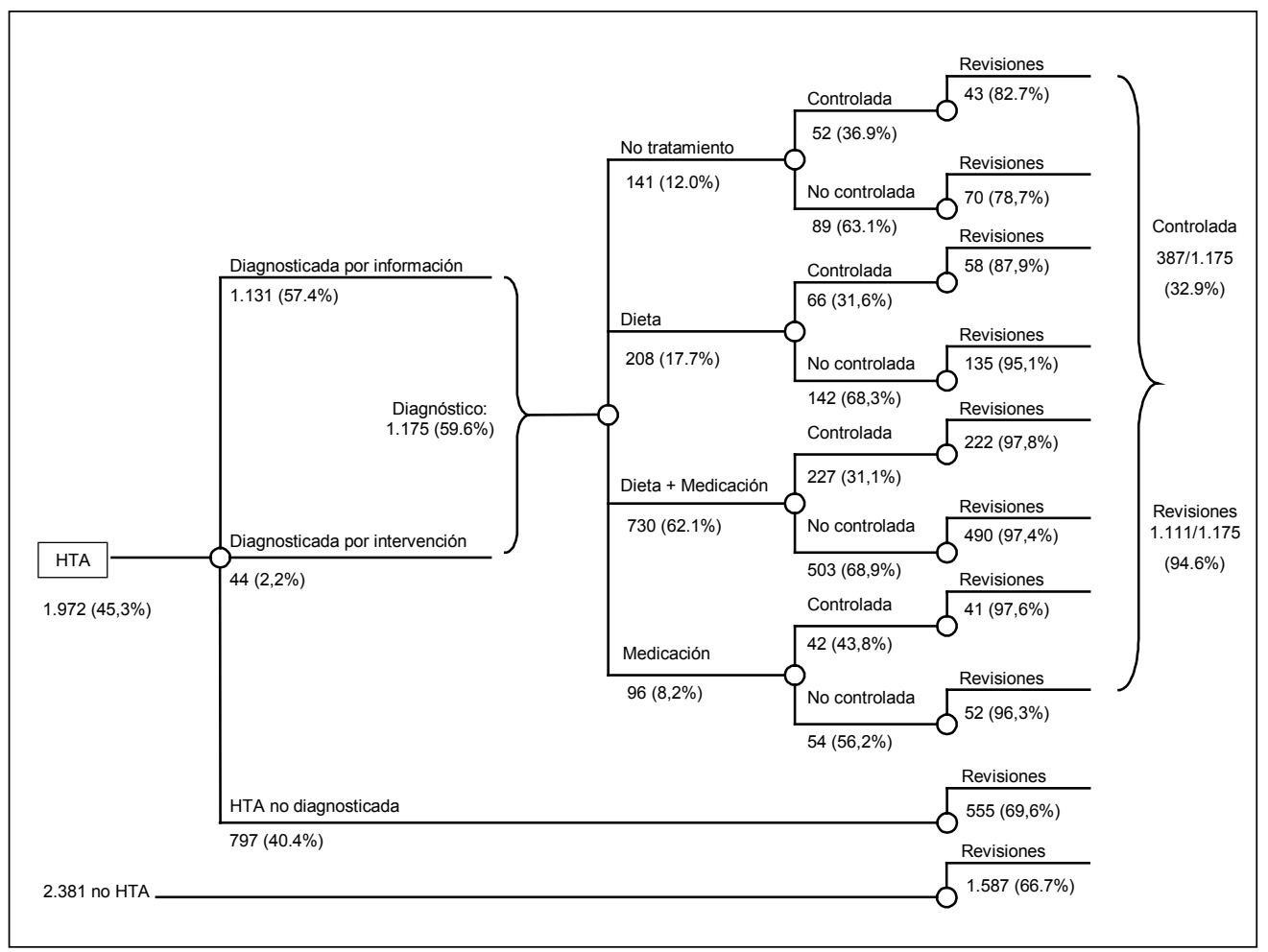

Figura 1. Hipertensión arterial: árbol resumen de porcentajes de diagnóstico, tratamiento, control y revisión en servicios sanitarios.

\section{Hipercolesterolemia}

Los resultados obtenidos de las intervenciones y controles sobre 4.168 personas fueron los siguientes:

La frecuencia de hipercolesterolemia fue en hombres el $41 \%$ y en mujeres el $37,3 \%$. Sin embargo, únicamente el $18,9 \%$ de los hombres y el $12,8 \%$ de las mujeres tienen el LDLc igual o superior a $160 \mathrm{mg} /$ dl y sólo alrededor del $8,5 \%$ tienen HDL inferiores a 40 y $50 \mathrm{mg} / \mathrm{dl}$ respectivamen$\mathrm{te}^{7}$.

El 85,6\% de ellos conocían su diagnóstico o estaban en tratamiento farmacológico con hipolipemiantes y el $14,5 \%$ fueron personas que no estaban diagnosticadas previamente. El 46,2\% de los diagnosticados había recibido sólo consejo dietético para su control, mientras que al $29,1 \%$ recibían intervención dietética y medicación. No refieren haber recibido ni consejo ni medicación el 18,8\%. El 5,9\% refieren únicamente la administración de fármacos para su tratamiento (Fig. 2).

De las hipercolesterolemias diagnosticadas previamente, estaban controlados en el momento del estudio con cifras inferiores a $250 \mathrm{mg} / \mathrm{dl}$ el $71 \%$. Este porcentaje alcanza el $95,1 \%$ en el grupo que refiere exclusivamente una intervención farmacológica.

El 78,6\% de los pacientes con hipercolesterolemia conocida se había realizado un análisis de control en el año anterior, en tanto que entre las personas no diagnosticadas se hicieron analítica el $45,5 \%$ cifra inferior a los no hipercolesterolemicos (53,3\%). 


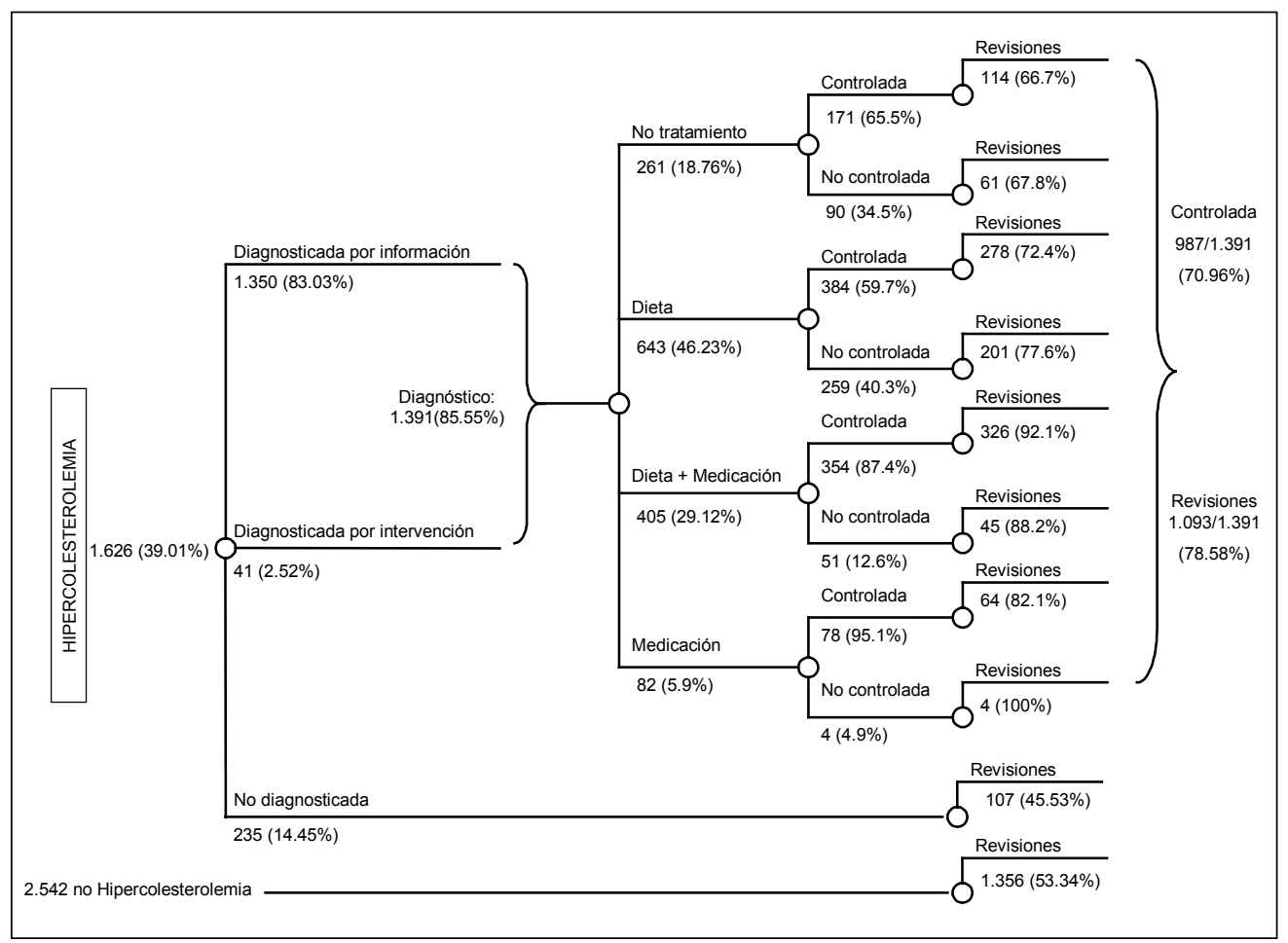

Figura 2. Hipercolesterolemia: árbol resumen de porcentajes de diagnóstico, tratamiento, control y revisión en servicios sanitarios.

\section{Diabetes y glucemia basal alterada}

Los resultados se obtuvieron sobre la analítica de 4.168 personas. La frecuencia de glucemia alterada y diabetes fue del $21,4 \%$. Dicho porcentaje fue más alto en varones que en mujeres ( $28,2 \%$ vs $15,7 \%$ ).

El $45,3 \%$ de las personas con glucemia alterada o con diabetes refirieron que el personal sanitario les había informado previamente de dicha situación, en tanto que el $0,6 \%$ no refieren haber sido informados, pero estaban siguiendo medicación específica para la diabetes. El resto de personas con glucemia alterada $(54,1 \%)$ no habían recibido información. De ellos el $44,7 \%$ eran personas con una glucemia entre 110 y 125 $\mathrm{mg} / \mathrm{dl}$ y el $9,4 \%$ eran posibles diabéticos con glucemia superior a $125 \mathrm{mg} / \mathrm{dl}$ (Fig. 3).

De las 409 personas diagnosticadas previamente de glucemia alterada o de diabetes, el 44,3\% refería haber recibido consejo dietético y medicación simultáneamente para su control, un 38,9 \% únicamente consejo dietético y un 3,2\% únicamente fármacos. El 13,7 \% informó que no ha recibido consejo dietético ni medicación para su control.

El 49,4\% de los diagnosticados estaban controlados (cifras iguales o inferiores a 130 $\mathrm{mg} / \mathrm{dl}$ ). Entre ellos, el 87,5\% no llevaban tratamiento específico, al $67,3 \%$ sólo se les había recomendado seguimiento dietético y el $22,1 \%$ recibieron consejo dietético y medicación. Entre los tratados sólo con medicación, las personas controladas fueron el $46,2 \%$.

Respecto a las revisiones, el $84,4 \%$ de las personas con glucemia alterada afirmó haberse realizado una analítica en el último año. Más de la mitad de las personas que no estaban previamente diagnosticadas se habían realizado analítica en el último año. El $52,4 \%$ de las personas con posible diabetes (glucemias superiores a $125 \mathrm{mg} / \mathrm{dl}$ en el estudio) y el $61,1 \%$ en los que se detectaba alteración de la glucemia basal referían disponer de analítica en el último año. 


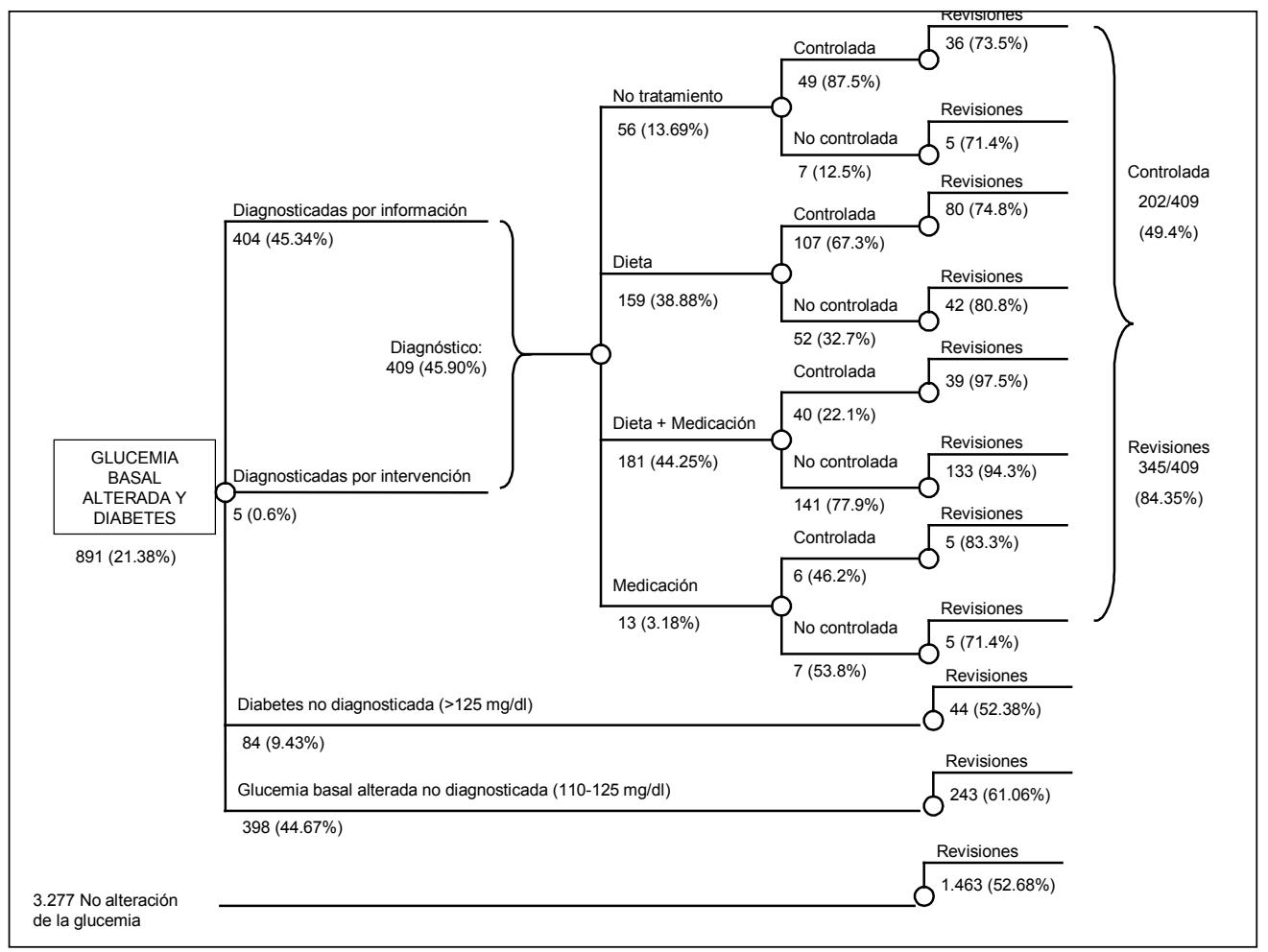

Figura 3. Glucemia basal alterada y diabetes: árbol resumen de porcentajes de diagnóstico, tratamiento, control y revisión en servicios sanitarios.

\section{Sobrepeso y obesidad}

De las 4.354 personas exploradas, la frecuencia de sobrepeso u obesidad fue del $65,5 \%$ : $77,3 \%$ en varones y $55,7 \%$ en mujeres El 39,5\% estaba diagnosticado previamente. De éstos, habían recibido consejo dietético el $66 \%$; dieta y medicación el 2,5\%; y únicamente medicación el $1,5 \%$. Un $30 \%$ refirieron no haber recibido ni consejo dietético ni tratamiento farmacológico (Fig. 4).

En el momento de la exploración, la frecuencia de obesidad, basada en el IMC, fue del $29,5 \%$ y de sobrepeso del $33 \%$. El $3,7 \%$ de los diagnosticados previamente como obesos, o con sobrepeso, estaban en situación de control con índice por debajo de niveles diagnósticos. El 76,3\% de las personas con sobrepeso u obesidad identificados refirieron haber realizado una medición de su peso en el último año.
Refirieron que se les había realizado previamente la medida de perímetro abdominal en un servicio sanitario el 9,5\% del total de la muestra, en tanto que en personas con sobrepeso u obesidad alcanzo el $17,1 \%$.

\section{DISCUSIÓN}

Al determinar la frecuencia de los factores de riesgo vascular e intentar comparar nuestros resultados con los de otros estudios similares, encontramos una serie de limitaciones: escasez de estudios epidemiológicos, utilización de diferentes cifras de normalidad, redefinición de criterios diagnósticos en los últimos años, variaciones en los criterios de inclusión o exclusión: p.e. de personas que reciben tratamiento farmacológico o bien pacientes que están en niveles controlados debido a la intervención terapéutica realizada. 


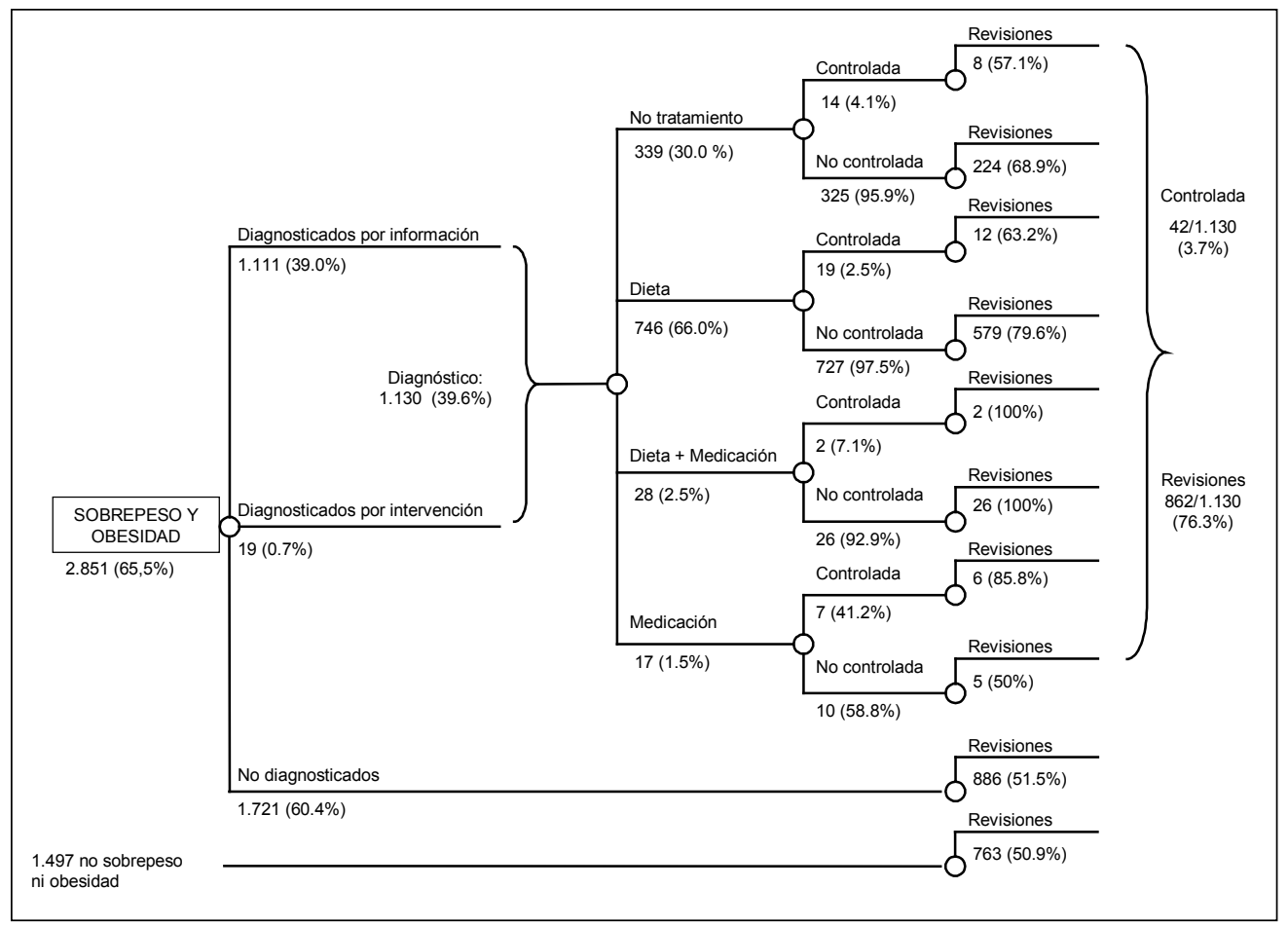

Figura 4. Sobrepeso y obesidad: árbol resumen de porcentajes de diagnóstico, tratamiento, control y revisión en servicios sanitarios.

En nuestro estudio, debido a que no se realizó confirmación diagnóstica posterior, al tratarse de un estudio transversal de punto de base poblacional, debemos aceptar una probable sobreestimación de las frecuencias observadas por estimar que la detección en personas asintomáticas produce falsos positivos. No así respecto a las intervenciones clínicas cuyos resultados se valoran sobre casos confirmados.

El análisis estadístico se desarrolla sobre frecuencias relativas ya que lo que se pretende es una información descriptiva de las intervenciones para conocimiento de los actores del sistema sanitario.

No obstante el carácter aleatorio y la magnitud de la muestra puede servir para hacer extrapolaciones a efectos clínicos.

\section{Hipertensión arterial}

La frecuencia de personas con cifras de hipertensión que, sin diagnóstico previo, detectamos en el estudio alcanza el 18,3\% (hipertensos "ocultos"). No obstante, el diagnóstico clínico correcto de hipertensión arterial precisa habitualmente de determinaciones múltiples obtenidas en momentos diferentes y durante cierto tiempo ${ }^{8}$. Estos criterios pueden justificar que no hayan sido identificados previamente como hipertensos algunas de las 555 personas que presentaban cifras de tensión arterial superiores a 140/90 $\mathrm{mmHg}$ en el momento del estudio, aunque referían que se les había realizado una toma de la tensión arterial en el último año.

En un estudio realizado en Baleares ${ }^{9}$ la frecuencia de HTA diagnosticada entre 3584 años fue de $25,6 \%$, y la oculta detectada el $22,2 \%$, alcanzando una tasa global de $47,8 \%$, resultando en su conjunto superior a la de Navarra.

La intervención farmacológica, con o sin consejo dietético (restricción de sal), alcanza al $70 \%$ de los hipertensos diag- 
nosticados, si bien el control de la tensión arterial en cifras inferiores a 140/90 $\mathrm{mmHg}$ se consigue en 1 de cada 3 hipertensos $(32,9 \%)$, siendo superior al $27,62 \%$ observado en Castilla-León ${ }^{10}$. Sin embargo, al igual que en el diagnóstico, la información para establecer el correcto o incorrecto control en su seguimiento debiera proceder también de determinaciones múltiples obtenidas en momentos diferentes y durante cierto tiempo.

La variabilidad en la frecuencia de personas controladas en función de la intervención realizada, deberemos interpretarla teniendo en cuenta la diferente severidad y evolución del diferente grado de hipertensión esperable en cada uno de los grupos de intervención observados.

La medida de la tensión arterial parece una exploración accesible y frecuente en nuestro medio, como lo demuestra que a más de $2 / 3$ partes de la población se la hayan tomado en el último año, porcentaje que se eleva hasta más del $95 \%$ en los pacientes con hipertensión detectada.

La frecuencia de un 18,3\% de hipertensión oculta y el hecho de que sólo el 32,9\% de hipertensos están controlados, indican la necesidad de intensificar las intervenciones en este factor de riesgo de gran importancia en la enfermedad cardio-cerebrovascular.

\section{Hipercolesterolemia}

A efectos de conocer la intervención clínica sobre el nivel en sangre de colesterol se ha utilizado un punto de corte diagnóstico definido en $250 \mathrm{mg} / \mathrm{dl}$, por ser el establecido por el Consenso para el Control de Hipercolesterolemia en España ${ }^{11}$. La proporción de hipercolesterolemia diagnosticada en Navarra del $39 \%$ es superior a la estimada en Baleares ${ }^{7}$, que fue de $24,2 \%$, si bien en este estudio no se incluyen aquellos pacientes hipercolesterolémicos en tratamiento y controlados con cifras inferiores a $250 \mathrm{mg} / \mathrm{dl}$ por lo que la diferencia deberá ser menor.

Más del 75\% de los pacientes identificados como hipercolesterolémicos refieren haber recibido consejo dietético. La prescripción de fármacos alcanza el $35 \%$ de los diagnosticados, lo que equivale al $12 \%$ de la población de 35 a 84 años. Es conocido el importante impacto económico que estos tratamientos tienen en nuestro sistema sanitario, siendo el tercer medicamento de mayor consumo en Navarra, detrás del omeprazol y del ácido acetilsalicílico en el año 2008, según datos propios del Servicio de Prestaciones del Servicio Navarro de Salud. Ello debe inducir la intensificación de la estrategia sanitaria orientada a obtener el máximo beneficio posible en la salud de nuestra población, mediante la indicación de tratamiento farmacológico en función del riesgo vascular global de cada persona. En prevención primaria, el riesgo debe ser estimado por sistemas de cálculo como REGICOR, SCORE o, esperamos que a corto plazo, el obtenido para la población de Navarra: "RICORNA»"12. La Guía Europea de Prevención Cardiovascular ${ }^{13}$ establece únicamente por encima de $320 \mathrm{mg} / \mathrm{dl} \mathrm{de}$ colesterol total (ó 240 de LDL) el manejo farmacológico con independencia de la estimación del riesgo global. El cociente colesterol total/colesterol HDL es también, en cualquier caso, un indicador que dados los valores elevados de HDL en nuestra población ${ }^{7}$ debe formar parte de la información a manejar por el clínico en la toma de decisión de intervención farmacológica en la hipercolesterolemia. La disponibilidad de los datos del Estudio de riesgo vascular de Navarra (RIVANA) permitirán profundizar más en la descripción de la situación e impacto de los lípidos en el riesgo vascular a nivel individual y poblacional y establecer predicciones y recomendaciones al respecto.

El 70\% de las personas que estaban previamente diagnosticadas presentaban niveles de colesterolemia inferiores a $250 \mathrm{mgr} /$ dl. Esta situación permite referirnos a una situación de control epidemiológico más elevado que para el resto de los factores de riesgo. Debemos aceptar, sin embargo, que siendo estrictos la definición de una situación de control clínico individual precisaría de una valoración de la adecuación de las cifras de colesterol a los niveles espe- 
cíficos de riesgo cardiovascular de las personas de la muestra. Es también elevado el número de pacientes con hipercolesterolemia que se realizan al menos una analítica anual ( $79 \%)$.

La frecuencia de revisiones analíticas de la población general, 53\% en el año anterior, justifica que la frecuencia de los hipercolesterolémicos "ocultos" en el momento del estudio sólo alcance el $6 \%$ de la muestra. Este dato, junto al anterior, nos confirma la sensibilidad social creada frente a este factor de riesgo, por encima de otros factores de mayor impacto.

\section{Diabetes y glucemia basal alterada}

La frecuencia de los trastornos de regulación de la glucosa ha sufrido en los últimos años cambios relevantes debido a nuevos criterios diagnósticos ${ }^{14,15}$ que junto a los valores de glucemia basal alterada aceptados en el "constructo" del Síndrome Metabólico, han dado lugar a una nueva perspectiva de salud pública. El diagnóstico previo de diabetes o de alteración de la glucemia fue del 10\% de la población. Además el 11,6\% presentaba cifras superiores a $110 \mathrm{mg} / \mathrm{dl}$ en el momento de la exploración por lo que podemos estimar en más del $21 \%$ la frecuencia de trastornos de regulación de la glucosa en las personas mayores de 35 años. De las personas detectadas con alteración de la glucosa el $2 \%$ eran posibles diabetes ocultas pendientes de confirmación y el $9,5 \%$ por glucemia basal alterada sin diabetes.

En el control del paciente diabético tienen relevancia, no sólo criterios metabólicos (glucemia basal, perfiles, hemoglobina glicada, etc.), sino también criterios de control de los otros factores de riesgo asociados, como la hipertensión arterial, el colesterol total o el HDL colesterol. En este trabajo, únicamente valoramos la situación de control en función de la analítica, con las limitaciones que ello supone. El 49,4\% de las personas estaban en situación de correcto control glucémico (glucemia basal igual o inferior a $130 \mathrm{mg} / \mathrm{dl}$ ), si bien es muy variable según el tipo de intervención.
El control es menor (22\%) en los pacientes intervenidos con consejo dietético y tratamiento farmacológico. Este dato podría explicarse razonablemente porque las personas en las que se realiza mayor intervención son los casos con mayor tiempo de evolución y mayor severidad del trastorno hidrocarbonado. No obstante, se evidencia una oportunidad de mejora en el control metabólico del paciente diabético.

\section{Sobrepeso y obesidad}

El sobrepeso y obesidad según el IMC afectan al $65,6 \%$ de la población, inferior al estudio de Baleares que fue el $67,1 \%{ }^{7}$. De ellos, las personas no identificadas previamente a la exploración superan el $60 \%$. En el $70 \%$ de las personas diagnosticadas previamente se realizaba intervención dietética, mientras que la intervención farmacológica no superaba, como era predecible, el $4 \%$ de los diagnosticados.

Más de la mitad de la población sin exceso de peso reconoce haberse pesado en el último año, elevándose hasta el 76,3\% en las personas con sobrepeso u obesidad diagnosticada. Sólo el 3,7\% de las personas que refieren haber sido diagnosticados previamente de sobrepeso u obesidad consiguen el control de peso con un IMC por debajo de $25 \mathrm{~kg} / \mathrm{m}^{2}$.

Al 9,5\% de las personas estudiadas les habían medido el perímetro abdominal. Este dato indica la incipiente introducción de este control en la práctica clínica en los últimos años. El perímetro abdominal es un indicador de obesidad central y depósito graso visceral como factor de riesgo más preciso que el IMC, motivo por lo que se incluye en la definición del Síndrome Metabólico. Impulsar la mayor frecuencia de su medición por los profesionales, en el contexto de la valoración de los factores de riesgo vascular y por los ciudadanos, en el contexto del autocuidado de su salud, debe seguir siendo objetivo de los sistemas de salud, reorientando la búsqueda del riesgo vascular más en el perímetro abdominal que en el IMC.

También la alta frecuencia de sobrepeso u obesidad según el IMC y la baja cifra 
de personas controladas, debe reorientar esta estrategia de intervención, complementando las actuaciones clínicas con acciones comunitarias de promoción de la salud y de fomento del autocuidado como la estrategia de salud más razonable y posiblemente de mayor impacto.

\section{CONCLUSIONES Y RECOMENDACIONES}

Las actuaciones preventivas poblacionales e individuales deben de estar orientadas por las características epidemiológicas de los factores de riesgo vascular de nuestra propia población. A partir de la información que nos proporciona el estudio en su conjunto y del análisis de los datos recogidos en este articulo, planteamos las siguientes conclusiones y recomendaciones:

1. El importante impacto epidemiológico en el riesgo vascular de nuestra población del conjunto: sobrepeso/ obesidad, hipertensión arterial e hiperglucemia requiere un esfuerzo renovado de los profesionales de salud en su identificación e intervención.

2. Es necesario mejorar la detección, intervención y control en la HTA. Creemos recomendable la automedición de la presión arterial para facilitar su seguimiento y mejorar la participación del paciente en su control.

3. La elevada prevalencia de la glucemia basal alterada y la oportunidad de intervención intensiva en el fomento de estilos de vida saludables (actividad física y alimentación) que su detección posibilita, hace que, junto con la propia diabetes, su identificación sea prioritaria en nuestra población.

4. Se debe orientar la intervención individual en la hipercolesterolemia teniendo en cuenta el conjunto del perfil lipídico y el riesgo vascular global. Esta intervención individualizada, y co-responsable con el paciente, debe ser la base del control de este factor de riesgo clásico. El índice $\mathrm{CT} / \mathrm{HDLc}$ tiene interés como indicador de exposición al riesgo, dado el alto nivel de HDLc en nuestra población.

5. La intervención individual y sobre todo poblacional para la prevención del sobrepeso-obesidad debe ser, junto a la del hábito tabáquico, prioritario para el conjunto del sistema de salud y de sus profesionales. La medición del perímetro abdominal debe ser una práctica habitual en la exploración clínica en las consultas de todos los profesionales sanitarios.

6. En un escenario de elevada prevalencia de factores de riesgo clásico en la población, creemos que la transferencia a los individuos de la responsabilidad de su cuidado debe orientar la estrategia de intervención individual para la mejora de los hábitos de vida saludables y para el propio control de los factores de riesgo vascular.

\section{BIBLIOGRAFÍA}

1. Mortalidad en Navarra 1996-2005. Boletín informativo Instituto de Salud Pública de Navarra. Mayo 2007; 44: 1-5.

2. Unal B, Critchley JA, Capewell S. Explaining the decline in coronary heart disease mortality in England and Wales, 1981-2000. Circulation 2004; 109: 1101-1107.

3. Ford ES, Ajani UA, Croft JB, Critchley JA, LABARTHE DR, KoTTKE TE et al. Explaining the decrease in U.S. Deaths from coronary disease, 1980-2000. N Engl J Med 2007; 356: 2388-2398.

4. Serrano M, Berjón J, Salaberri A, Amézqueta C. Riesgo Cardiovascular. Evidencias que orientan la actuación clínica. Gobierno de Navarra. An Sist Sanit Navar 2003: Monografía nº 2: 1-117.

5. Viñes JJ, Díez J, Guembe MJ, GonzÁlez P, AmÉzQueTA C, BARBA J et al. Estudio de riesgo vascular en Navarra: objetivos y diseño. Prevalencia del síndrome metabólico y de los factores mayores de riesgo vascular. An Sist Sanit Navar 2007; 30: 113-124.

6. Guembe MJ. Prevalencia por 100 de factores de riesgo en Navarra 1993: Bol Salud Pub Nav 1994; 7: 2-8.

7. Viñes JJ, Díez J, Guembe MJ, González D, AmézQUETA C, BARBA J et al. Estudio de riesgo vascu- 
lar en Navarra: objetivo y diseño. Prevalencia del síndrome metabólico y de los factores mayores de riesgo vascular. An Sist Sanit Navar 2007; 30: 113-124.

8. Guidelines for the Management of Arterial Hypertension. The Task Force for the Management of Arterial Hypertension of the European Society of Hypertension (ESH) and of the European Society of Cardiology (ESC). J Hypertens 2007; 25: 1105-1187.

9. Rigo Corratalá F, Guillén Frontera J, Llobera Cánaves J, Rodríguez Ruz T, Borrás Bosch I, Fuentespina Vidal E. Prevalencia de factores de riesgo cardiovascular en las Islas Baleares (estudio CORSAIB). Rev Esp Cardiol 2005; 58: 1411-1419.

10. Riesgo de Enfermedad Cardiovascular en Castilla y León. 2005. Consejería de Sanidad. Junta de Castilla y León.

11. Ministerio de Sanidad y Consumo. Sociedad Española de Cardiología. Consenso para el Control de la Colesterolemia en España. Madrid: MSC; 1990.
12. González P, Moreno C, Guembe MJ, Vila J, Elosúa R, VIÑES JJ et al. Estimación del riesgo de enfermedad coronaria en la población Navarra (RICORNA). Pamplona: Gobierno de Navarra An Sist Sanit Navar 2008; En: Monografía n⿳ำ 4: Riesgo Vascular en Navarra: 61-68.

13. Guía europea de prevención cardiovascular en la práctica clínica. Tercer grupo de trabajo de las sociedades europeas y otras sociedades sobre la prevención cardiovascular en la práctica clínica. Eur Heart J 2003; 24: 16011610.

14. Expert committee on the diagnosis and classification of diabetes mellitus. Report of the expert committee on the diagnosis and classification of diabetes mellitus. Diabetes Care 1997; 20: 1183-1197.

15. World Health Organization. Definition, diagnosis and classification of diabetes mellitus and its complications: Report of a WHO Consultation. Part 1. Diagnosis and classification of diabetes mellitus. Geneva: World Health Organization, 1999. 